\title{
层状双金属氢氧化物及复合材料对放射性元素铀的吸附及机理研究
}

\author{
王宁 ${ }^{a, b}$ 庞宏伟 ${ }^{b}$ 于淑君 ${ }^{b}$ 顾鹏程 ${ }^{b}$ \\ 宋爽 ${ }^{b}$ 王宏青*, $a$ 王祥科*, \\ $\left({ }^{a}\right.$ 南华大学 化学化工学院 衡阳 421000) \\ ( $b$ 华北电力大学 环境科学与工程学院 北京 102206)
}

\begin{abstract}
摘要 随着核工业的快速发展, 大量放射性元素铀被排放到环境中, 造成严重的环境污染并给人类健康带来重大危害. 层状双金属氢氧化物(LDHs)因其具有比表面积大、离子交换能力强以及独特的纳米结构等优点, 在铀酰离子的去除及 环境水污染处理方面展现出巨大潜力. 同时, 将层状双金属氢氧化物进行改性可大大增加活性位点, 进一步提高材料 对放射性元素铀的吸附性能. 详细介绍了层状双金属氢氧化物及复合材料的制备及改性方法, 通过光谱分析技术阐述 了层状双金属氢氧化物对环境中铀酰离子的吸附效果以及作用机理. 最后, 对层状双金属氢氧化物在治理水污染中的 应用前景给出个人见解, 以期为今后的环境治理工作的深入研究和实际应用提供参考依据.

关键词 层状双金属氢氧化物; 复合材料; 铀; 环境污染; 吸附
\end{abstract}

\section{Investigation of Adsorption Mechanism of Layered Double Hydroxides and Their Composites on Radioactive Uranium: A Review}

\author{
Wang, Ning ${ }^{a, b} \quad$ Pang, Hongwei ${ }^{b} \quad$ Yu, Shujun ${ }^{b} \quad$ Gu, Pengcheng ${ }^{b}$ \\ Song, Shuang ${ }^{b} \quad$ Wang, Hongqing ${ }^{*, a}$ Wang, Xiangke ${ }^{*, b}$ \\ $\left({ }^{a}\right.$ School of Chemistry and Chemical Engineering, University of South China, Hengyang 421000) \\ ( ${ }^{b}$ College of Environmental Science and Engineering, North China Electric Power University, Beijing 102206)
}

\begin{abstract}
With the rapid development of nuclear industry, nuclear energy, as a kind of low-carbon energy, has been widely used in the world. However, in the development and application of nuclear energy, a large amount of radionuclides, especially the radioactive uranium, have been inevitably discharged into the environment, causing serious environmental pollution and having great harm to human health. Layered double hydroxides (LDHs) have become the excellent adsorbents in environmental pollution treatments due to easy preparation, large specific surface area, the unique nanostructure and excellent ion exchange capacity. Hence, the preparation of layered double hydroxides and their composites for the efficient removal of radioactive uranium is one of the hot issues in the field of environmental science, which include coprecipitation, ion exchange, hydrothermal method, the urea hydrolysis method, aerogel, microwave-crystallization and separate nucleation/crystallization isolation method. Besides the aforementioned methods, other reported synthesis methods of LDHs include the secondary intercalation method (an intercalation method involving dissolution and the re-coprecipitation method), reconstruction method based on the "memory effect", $\mathrm{N}_{2}$ protection synthesis, mechanochemical synthesis, surface synthesis, template synthesis, and others. The modification methods of layered double hydroxides can be divided into calcination, intercalation and compounding method, which significantly increase the active sites and further improve the adsorption performance of the materials to radioactive uranium. In addition, the adsorption mechanism has been thoroughly investigated with spectroscopic analysis techniques such as Fourier transformed infrared spectroscopy (FT-IR), X-ray diffraction (XRD), X-ray photoelectron spectroscopy (XPS) and Extended X-ray absorption fine structure (EXAFS). In conclusion, the review briefly discuss the application prospects of layered double hydroxides and their composites in the treatment of water pollution, which provide definitive reference values for the further research and practical application of environmental management in the future.
\end{abstract}

Keywords layered double hydroxides; composites; U(VI); environmental pollution; adsorption

\section{1 引言}

随着核工业的快速发展, 核能作为一种低碳能源,
得到全球范围内的广泛使用 ${ }^{[1 \sim 3]}$. 但在核能的开发及应 用过程中，大量放射性元素被排放到环境中，造成了严 重的环境污染并给人类健康带来重大危害 ${ }^{[4 \sim 6]}$. 铀

\footnotetext{
* E-mail: HQWang2001cn@126.com. (Wang Hongqing), Tel.: 0734-8281676; xkwang@ncepu.edu.cn. (Wang Xiangke), Tel.: 010-61772890 Received September 26, 2018; published October 10, 2018.

Project supported by the National Natural Science Foundation of China (No. 21577032) and the Science Challenge Project (No. TZ201604).

项目受国家自然科学基金(No. 21577032)和挑战计划(No. TZ201604)资助.
} 
$(\mathrm{U}(\mathrm{VI}))$ 是核废液中主要的放射性污染物之一，半衰期 长, 能长期稳定地存在于土壤和水体中, 具有很强的化 学毒性和放射性, 长期遭受铀辐射会使皮肤发生癌变, 心脏能力衰竭甚至死亡 ${ }^{[7 ~ 9]}$. 因此, 在废水排入环境之 前, 去除放射性元素铀对于环境保护及人类健康是至关 重要的.

目前, 从水溶液中去除放射性元素铀的方法主要有 沉淀法、催化法、过滤、膜分离法、离子交换法以及吸 附等 ${ }^{[10 ~ 14]}$. 其中吸附技术由于操作简单、经济成本低和 实用性强等优点受到广泛关注, 尤其在工业废水治理方 面, 吸附成为去除水溶液中污染物最常见和最高效的方 法之一[15 17]. 常见的吸附材料有生物质材料、碳基纳米 材料、黏土矿物材料、磁性材料以及层状双金属氢氧化 物材料等. 近年来, 以壳聚糖为典型代表的生物质材料 被广泛用于去除环境污水中的放射性元素铀, 但是吸附 效率极易受到温度、离子强度和溶液 $\mathrm{pH}$ 等环境因素的 限制. 碳基纳米材料也被广泛用作吸附剂, 如碳纳米管 材料和氧化石墨烯(GO)材料以其对污染物吸附量高和 去除速率快等优点被广泛应用 ${ }^{[18,19]}$, 但存在成本高、制 备复杂、易产生二次污染等局限性. 以蒙脱土为代表的 黏土矿物材料由于价格低廉、来源广泛等优点也被广泛 用于废水处理领域, 但实际应用中对污染物处理效率较 低 ${ }^{[20]}$. 另外, 以四氧化三铁为代表的磁性材料因性质稳 定、容易分离等优势被认为是处理铀酰离子的潜在材 料 ${ }^{[21]}$, 但其吸附效率低, 实际应用具有一定局限性. 因 此，优良的吸附材料要同时考虑吸附效率、经济成本、 环境稳定性以及实际应用性等多方面因素, 以求得经济 效益和环境效益的最大化.

层状双金属氢氧化物(LDHs) 是一种薄层状无机材 料, 别名类水滑石, 结构类似于水镁石 $\mathrm{Mg}(\mathrm{OH})_{2}$, 由 $\mathrm{MgO}_{6}$ 八面体共用棱形成单元层 ${ }^{[22]}$. LDHs 的化学组成可 用 $\left[\mathrm{M}^{\mathrm{II}}{ }_{1-x} \mathrm{M}^{\mathrm{III}}{ }_{x}(\mathrm{OH})_{2}\right]^{x+}\left(\mathrm{A}^{n-}\right)_{x / n}{ }^{x-} \cdot m \mathrm{H}_{2} \mathrm{O}$ 表示. 其中, $\mathrm{M}^{\mathrm{II}}$ 和 $\mathrm{M}^{\mathrm{III}}$ 分别代表二价金属阳离子(如 $\mathrm{Mg}(\mathrm{II}) 、 \mathrm{Ni}(\mathrm{II}) 、 \mathrm{Fe}(\mathrm{II}) 、$ $\mathrm{Ca}(\mathrm{II}) 、 \mathrm{Zn}(\mathrm{II}) 、 \mathrm{Cu}(\mathrm{II})$ 等)和三价金属阳离子(如 $\mathrm{Al}(\mathrm{III})$ 、 $\mathrm{Fe}(\mathrm{III}) 、 \mathrm{Mn}(\mathrm{III}) 、 \mathrm{La}(\mathrm{III})$ 等), $\mathrm{A}^{n-}$ 代表层间阴离子(如 $\mathrm{CO}_{3}{ }^{2-} 、 \mathrm{NO}_{3}{ }^{-} 、 \mathrm{Cl}^{-}$等 $), x$ 是 $\mathrm{M}^{\mathrm{III}} /\left(\mathrm{M}^{\mathrm{II}}+\mathrm{M}^{\mathrm{III}}\right)$ 的物质的量 比, $m$ 是共混水的物质的量 ${ }^{[23 ~ 26]}$. 图 1(a) 是 Mg-Al-LDHs 的样貌形态图, 通常呈白色粉末状. 图 1(b) 是 Mg-Al-LDHs 的结构示意图, 位于层上的 $\mathrm{Mg}(\mathrm{II})$ 可在一 定的范围内被 $\mathrm{Al}$ (III) 同晶取代, 使得层板带正电荷, 层 间有可交换的 $\mathrm{CO}_{3}{ }^{2-}$ 与层板上的正电荷平衡, 使得水滑 石的整体结构呈电中性. 由于层板和层间阴离子通过氢 键连接, 使得 LDHs 层间阴离子具有可交换性. 此外, 在 LDHs 中存在层间水这些水分子可以在不破坏层状结 构条件下除去. 由于 LDHs 不仅来源广泛、比表面积大， 而且具有优越的离子交换能力以及高效的分散性 ${ }^{[27 ~ 29]}$, 因此被广泛应用于高效吸附剂、离子交换器以及光催化 领域 ${ }^{[30 ~ 33]}$. 另外, 可通过煅烧、插层及复合改性等方法
对 LDHs 进行处理，增加材料表面官能团或者引入具有 亲、疏水端的有机物，从而极大地提高吸附剂材料对 $\mathrm{U}(\mathrm{VI})$ 的吸附容量 ${ }^{[34]}$.

LDHs 材料在治理铀污染方面有着其他吸附材料不 可比拟的优势. 深入研究 LDHs 材料和放射性元素铀之 间的相互作用机理，将 LDHs 材料更为高效地应用在环 境污染治理方面有着极其深远的意义. 鉴于此, 本文综 合论述了近年来 LDHs 及其复合材料对于去除放射性元 素铀的相关研究和进展, 系统地归纳了 LDHs 材料的制 备和改性方法以及对 U(VI)的去除效果和去除机理，并 对 LDHs 材料的应用前景进行了展望.

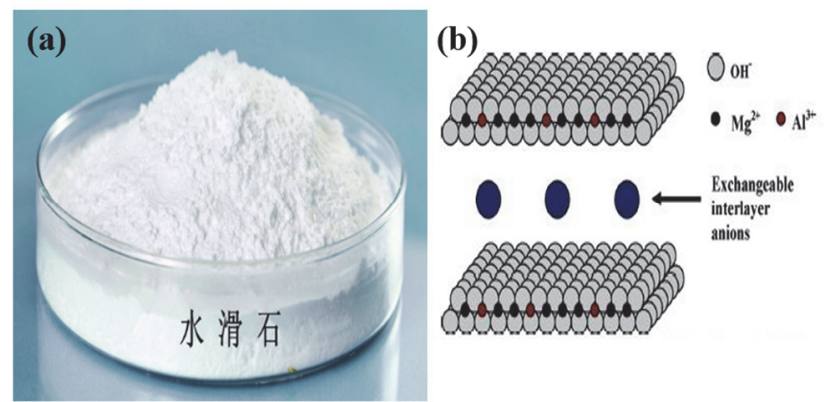

图 1 (a) Mg-Al-LDHs 的形态样貌图; (b) Mg-Al-LDHs 的结构示意图 Figure 1 (a) The morphology of Mg-Al-LDHs; (b) schematic representation of Mg-Al-LDHs

\section{2 层状双金属氢氧化物的制备}

LDHs 材料的制备相对简单, 通常在实验室即可合 成(由于实验设备及制备条件等因素的限制，产品纯度 可能受到一定影响). 常见的制备方法主要有共沉淀法、 离子交换法、水热法、尿素水解法和机械化学合成法等, 下面进行详细介绍 ${ }^{[35,36]}$.

\section{1 共沉淀法}

共沉淀法是制备 LDHs 材料最简单、最常用的方法. 在此方法中, 含有二价、三价金属离子以及 $\mathrm{CO}_{3}{ }^{2-}$ 等阴 离子的水溶液作为前体溶液, $\mathrm{Mg}(\mathrm{II})$ 和 $\mathrm{Al}(\mathrm{III})$ 作为常用 的金属前体物. 为了保证两种或多种阳离子同时沉淀, 需要在过饱和情况下进行合成, 因此共沉淀法又分为低 饱和共沉淀法和高饱和共沉淀法. 低饱和共沉淀法是按 照一定的比例，将金属硝酸盐溶液配成一定浓度的混合 盐溶液(SolS), 将 $\mathrm{NaOH}$ 和 $\mathrm{Na}_{2} \mathrm{CO}_{3}$ 按照一定比例配成混 合碱溶液(SolB), 在容器中预先装入一定量的蒸馏水, 加热至一定的温度, 将 SolS 和 SolB 按一定的滴速同时 滴入蒸馏水中, 维持反应体系的 $\mathrm{pH}$ 为一恒定值, 剧烈 搅拌. 滴定完毕后, 继续搅拌陈化, 洗涤后烘干得到产 物. 其中镁盐和铝盐可以采用硝酸盐、硫酸盐、氯化物 等, 碱可以采用氢氧化钠、氢氧化钾、氨水等，碳酸盐 可以采用碳酸钠、碳酸钾等，也可以采用尿素代替碱和 碳酸盐. 高过饱和共沉淀法, 即将 SolS 和 SolB 各自预 先加热至反应温度, 然后快速将两种溶液同时倒入装有 
预先加热到和该溶液具有相同温度的二次蒸馏水的容 器中, 剧烈搅拌, 最后洗涤烘干得到产物 ${ }^{[37]}$. 值得注意 的是, 高/低饱和共沉淀过后都需要对材料进行热处理 来提高水滑石的产量和结晶度. 例如, Chen 等 ${ }^{[38]}$ 通过共 沉淀法及水热老化处理成功合成了 $\mathrm{Ca}-\mathrm{Al}$-LDHs 功能化 碳纳米管复合材料(Ca-Al-LDH@CNTs), 整个合成路线 如图 2 所示.

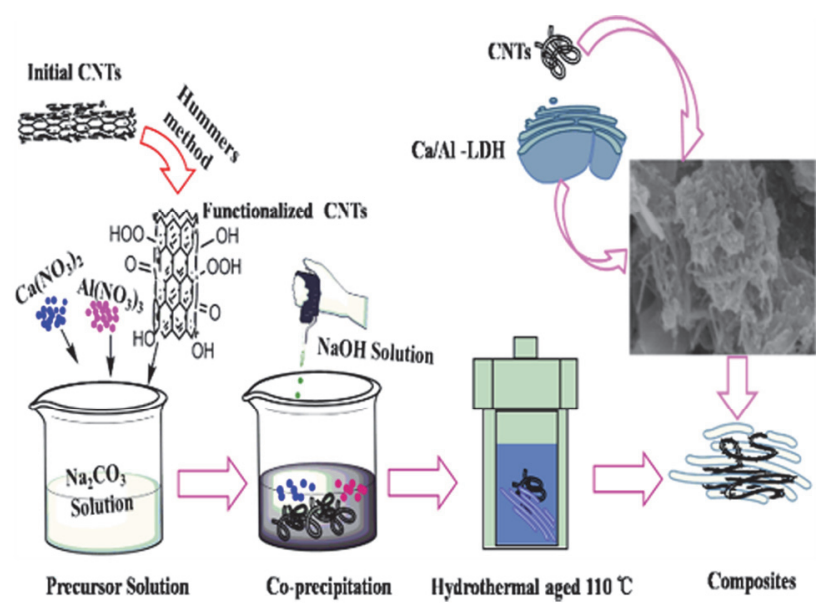

图 2 Ca-Al-LDH@CNTs 复合材料的合成路线图 ${ }^{[38]}$

Figure 2 Proposed synthetic routes for the synthesis of Ca-AlLDH@CNTs composites ${ }^{[38]}$

\section{2 离子交换法}

当金属离子在碱性介质中不稳定，或当阴离子 $\mathrm{A}^{n-}$ 没有可溶性的 $\mathrm{M}^{\mathrm{II}}$ 和 $\mathrm{M}^{\mathrm{III}}$ 盐类, 共沉淀法无法进行时, 可 采用离子交换法制备层状双金属氢氧化物. 该法是从给 定的 LDHs 出发, 通过溶液中某种阴离子对原有阴离子 的交换作用, 形成新的相 ${ }^{[39]}$. 然而在 LDHs 材料上, 直 接用大体积无机阴离子通过离子交换法制备很困难，一 般先用大体积有机阴离子把层间撑开, 然后用无机阴离 子交换制得样品.

\section{3 水热法}

当与 LDHs 亲和度低的有机客体需要插入到 LDHs 层间或者共沉淀法和离子交换法不适用时, 此时采用水 热法. 当不溶的 Mg-Al-LDHs 被用作前体物, 在水热情 况下只有特定的有机阴离子才能够占据夹层内空 间 ${ }^{[40,41]}$. 例如, Wang 等 ${ }^{[42]}$ 将尿素加入到分别含有二价金 属硝酸盐 $\left(\mathrm{Mg}\left(\mathrm{NO}_{3}\right)_{2}, \mathrm{Ni}\left(\mathrm{NO}_{3}\right)_{2}, \mathrm{Zn}\left(\mathrm{NO}_{3}\right)_{2}\right)$ 和 $\mathrm{Al}\left(\mathrm{NO}_{3}\right)_{3}$ 混 合溶液中, $\mathrm{M}^{\mathrm{II}} / \mathrm{Al}^{\mathrm{III}} /$ 尿素物质的量比例设为 $3: 1: 15$, 成功采用水热法制备出 Mg-Al-LDHs, Ni-Al-LDHs 和 Zn-Al-LDHs.

\section{4 尿素水解法}

Jaiswal 和 $\mathrm{Wu}$ 等 ${ }^{[43,44]}$ 曾经报道过关于尿素水解法制 备 LDHs 的相关研究. 尿素具有较高的水溶性以及易于 调节的水解速率等特性, 因此当有适当阴离子存在时常 作为氢氧化物或不溶性盐来沉淀溶液中的金属离子. 在 相对较短时间内采用尿素法制备出具有良好晶体品质
的 LDHs 的最佳参数是, 将固体尿素溶解在 $0.5 \mathrm{~mol} / \mathrm{L}$ 特 定的金属氯化物中，尿素与金属离子物质的量比为 3.3 . 此方法制备出的 LDHs 尺寸均匀, 具有良好的六边形薄 片形态. 另外，六次甲基四胺(HMT)也常代替尿素用于 LDHs 材料的合成.

\section{5 成核晶化隔离法}

成核/晶化隔离法, 是将 SolS 和 SolB 迅速于全返混 旋转液膜成核反应器中混合, 剧烈循环搅拌几分钟, 然 后将浆液于一定温度下晶化. 采用该反应器实现盐液与 碱液的共沉淀反应, 通过控制反应器转子线速 $\left(5 \mathrm{~m} \cdot \mathrm{s}^{-1}\right)$ 可使反应物瞬间充分接触、碰撞，成核反应瞬间完成， 同时成核混合物在胶体磨中受到的极端力量能够阻止 核的聚集，导致晶体成核粒径小且尺寸均匀. 当最终的 混合物经历分离老化过程后, 最终形成结构良好的结 晶.

\section{6 机械化学合成法}

机械化学合成法即在高能球磨机中, 通过直接研磨 金属盐和碱等前驱体来制备 LDHs. 例如, Yu 等 ${ }^{[45]}$ 以 $\mathrm{Mg}(\mathrm{OH})_{2} 、 \mathrm{Al}(\mathrm{OH})_{3}$ 和 $\mathrm{NaHCO}_{3}$ 为起始反应物, 采用机械 化学合成法, 制备出粒子平均晶粒度约 $20 \mathrm{~nm}$ 的高结晶 度单相纳米晶 Mg-Al-LDHs. 在传统方法的基础上对 LDHs 材料的制备进行了一定的创新. 然而, 这种方法 存在一定局限性, 即在 LDHs 制备过程中, 会掺杂 $\mathrm{Al}(\mathrm{OH})_{3}$ 杂质, 从而影响材料的纯度.

\section{7 气凝胶法}

气凝胶法一般用于纳米级 LDHs 的合成，这种方法 的主要特征是在甲醇-甲苯混合液中, 水解作用、凝胶 化、热液处理以及最后的溶剂进行超临界干燥时, 金属 有机前体会发生均质化，因此制备出的 LDHs 颗粒微小 且比表面积大.

除以上方法外，其他制备 LDHs 的方法有二次插入 法(一种涉及溶解和再共沉淀的插入方法)、记忆效应重 建法、微波晶化法、 $\mathrm{N}_{2}$ 保护合成法、表面合成法以及模 板合成法等 ${ }^{[46 ~ 49]}$.

\section{3 层状双金属氢氧化物的改性方法}

过去几十年, 研究人员对层状双金属氢氧化物在环 境污染物处理领域的应用进行了大量研究. 尽管 LDHs 材料能够高效去除阴离子污染物, 但是对于阳离子污染 物的去除效率较低, 且单纯的 LDHs 存在官能团少、耐 酸性差等缺陷，这严重制约了其在处理环境污水方面的 应用, 因此越来越多的研究人员试图通过对 LDHs 进行 表面改性如煅烧 ${ }^{[50,51]}$ 、插层 ${ }^{[52 ~ 54]}$ 和复合改性 ${ }^{[21,55 ~ 57]}$ 等方 法来提高 LDHs 材料的层间距、比表面积和表面官能团 等, 进而增加其与铀酰离子之间的作用位点, 提高吸附 性能. 


\section{1 煅烧法}

煅烧是一种有效改性 LDHs 的方法, 共包括脱水 (I)、脱羟基(II)、阴离子分解(III) 以及氧化物改性(IV)四 个过程. LDHs 经过高温㷽烧后 $\left(T<600{ }^{\circ} \mathrm{C}\right)$, 由六边形 形状转为无定形状态, 层间结构被破坏, 因此有利于提 高衍生物的特定表面积. 此外, 夹层中的阴离子和水分 子被去除以及金属含氧功能基团的增加，均为污染物的 捕获提供了更多的结合位点, 因此大大提高了 LDHs 对 污染物的吸附性能 ${ }^{[51]}$. 例如, Zou 等 ${ }^{[58]}$ 成功合成了 $\mathrm{Ca}$ Mg-Al-LDH 以及其经高温蚌烧得到的一系列 Ca-Mg$\mathrm{Al}-\mathrm{LDO}_{x}$ 材料 ( $x$ 代表不同的㷽烧温度). 随着㷽烧温度的 增加, 材料的团聚现象明显得到缓解, 进而提高了材料 的表面配位能力. 由实验结果得出 Ca-Mg-Al- $\mathrm{LDO}_{500}$ 材 料对水溶液中 $\mathrm{U}(\mathrm{VI})$ 的吸附量可达 $486.8 \mathrm{mg} / \mathrm{g}$, 吸附效 果远高于煅烧前 Ca-Mg-Al-LDH 的吸附量 $(132.5 \mathrm{mg} / \mathrm{g}$ ).

\section{2 插层法}

插层法是为了增大层间距、引入携带某些特异性官 能团的物质或离子或者引入具有特殊性质(如超疏水性) 的物质. 例如, Wei 等 ${ }^{[59]}$ 通过精确控制条件可将具有不 同层间距 $(1.03$ 和 $1.44 \mathrm{~nm})$ 的单价或双价的 $L$-半胱氨酸 阴离子插入到 Mg-Al-LDHs 材料中, 制备出结晶良好的 ( $L$-CysH-LDHs) 材料. $\mathrm{Yu}$ 等 ${ }^{[60]}$ 通过水热法将芳香酸阴离 子(对苯二甲酸和均苯四羧酸) 插入到水滑石中, 其合成 示意图如图 3(a)所示, 并系统研究了该材料在水溶液中 对苯胺的去除行为. 由于层间距增大以及含氧官能团的 增多, 改性后材料对苯胺的去除量为原始 LDHs 的 2 3 倍.

\section{3 复合改性}

近年来, 制备 LDHs 与碳基纳米材料、磁性材料以 及甘油等物质的复合材料一直是研究热点, 并取得了一 定研究成果.

由于碳基纳米材料具有吸附效果好, 去除速率快等 优点, 使二者的复合材料在环境污染处理方面具有巨大 潜力. 例如, Wen 等 ${ }^{[6]}$ 通过一步水热合成法制备得到的 LDHs 和 $\mathrm{GO}$ 的复合材料用于水溶液中 $\mathrm{As}(\mathrm{V})$ 的去除. 图 3 (b) 是 Mg-Al-LDHs 的透射电镜图, 展现出典型的六边 形结构, 图 3(c)是 $\mathrm{LDHs} / \mathrm{GO} 2$ 复合物的透射电镜图, 从 该图中可以明显看出皱纹状 $\mathrm{GO}$ 附在 $\mathrm{LDHs}$ 纳米片上, 意味着氧化石墨烯与水滑石复合材料的成功合成. Yu 等 ${ }^{[16]}$ 研究了氧化石墨烯复合 LDHs 材料从环境水溶液中 分离去除 $\mathrm{U}(\mathrm{VI})$, 实验结果拟合 Langmuir 等温线模型且 最大吸附量可达 $160.0 \mathrm{mg} / \mathrm{g}$, 相比 LDHs 单体(69.0 $\mathrm{mg} / \mathrm{g})$ 和 GO $(92.0 \mathrm{mg} / \mathrm{g})$, 该复合物对 U(VI)的吸附性能 显著提高.

四氧化三铁 $\left(\mathrm{Fe}_{3} \mathrm{O}_{4}\right)$ 由于具有独特的磁分离特性, 在 生物靶向材料、磁性流体、静电复印显影剂以及高梯度 磁性分离器等领域具有较好的应用前景 ${ }^{[62 ~ 65]}$. 因此, 将
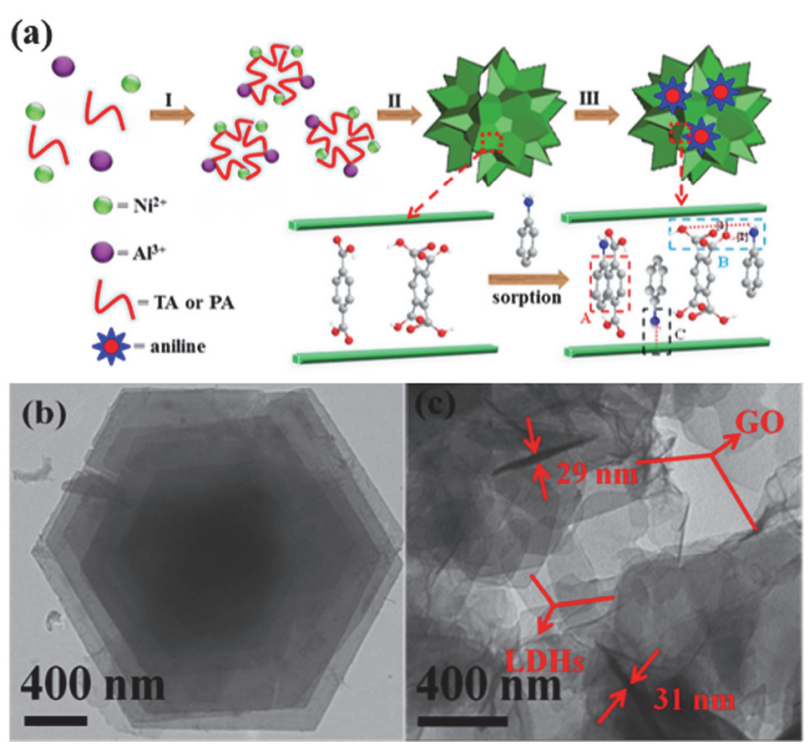

图 3 (a) LDH, TAL 和 PAL 材料的合成方法示意图以及与苯胺的作用 机理图(I: Ni(II)/Al(III)-TA/PA 复合物的形成; II: 水热法; III: 与苯胺 的相互作用) (原子代码: 灰 $-\mathrm{C}$, 红 $-\mathrm{O}$, 蓝 $-\mathrm{N}$, 白 $-\mathrm{H}$; 三种机 理: $\pi-\pi$ 作用, 氢键和静电作用) ${ }^{[60]}$; LDHs (b) 和 $\mathrm{LDHs} / \mathrm{GO} 2$ (c) 的透射 电镜图 ${ }^{[61]}$

Figure 3 (a) Schematic illustration of the approach used for the preparation of the materials (I: formation of $\mathrm{Ni}(\mathrm{II}) / \mathrm{Al}(\mathrm{III})-\mathrm{TA} / \mathrm{PA}$ complex; II: hydrothermal treatment; III: interaction with aniline) and the proposed mechanisms of aniline onto LDH, TAL and PAL (Color code by atom: gray carbon, red oxygen, blue nitrogen, white hydrogen. The three interactions are $\pi-\pi$ interaction, hydrogen bond and electrostatic interaction ${ }^{[60]}$; TEM images of LDHs (b) and LDHs/GO2 (c) $)^{[61]}$

四氧化三铁与 LDHs 材料进行复合, 不仅保留了 LDHs 自身的吸附能力，同时赋予了复合材料磁性特质，更易 从环境水溶液中分离出来. 如图 4 所示, Yang 等 ${ }^{[6]}$ 成功 制备了多巴胺和水滑石包裏的 $\mathrm{Fe}_{3} \mathrm{O}_{4}$ 纳米颗粒 $\left(\mathrm{Fe}_{3} \mathrm{O}_{4} @ \mathrm{PDA} @ \mathrm{LDHs}\right)$ 并将其用于 $\mathrm{U}(\mathrm{VI})$ 的高效去除. 如 图 4(a) $\sim$ 4(d) 所示, $\mathrm{Fe}_{3} \mathrm{O}_{4}, \mathrm{Fe}_{3} \mathrm{O}_{4} @ \mathrm{LDHs}$ 和 $\mathrm{Fe}_{3} \mathrm{O}_{4} @ \mathrm{PDA} @ \mathrm{LDHs}$, 形貌为球形, $\mathrm{Fe}_{3} \mathrm{O}_{4} @ \mathrm{PDA} @ \mathrm{LDHs}$ 呈现出明显的壳核结构, 并具有良好的分散性. 图 4(e) 是三种材料的磁滞回线, 饱和磁化量分别是 62.02 、

20.88 和 $16.49 \mathrm{emu} / \mathrm{g}$. 尽管磁性有所下降, 但是复合材 料仍然能够在外界磁场下快速固液分离. 五个循环实验 后, $\mathrm{Fe}_{3} \mathrm{O}_{4} @ \mathrm{PDA} @$ LDHs 对 U(VI)的去除率仍高于 85\% (图 4(f)). 另外, Sheng 等 ${ }^{[67]}$ 采用共沉淀法制备的纳米零 价铁与 LDHs 复合材料(NZVI/LDHs) 比表面积为 52.4 $\mathrm{m}^{2} / \mathrm{g}$, 在 $\mathrm{pH}=6.5$ 时对 $\operatorname{Re}(\mathrm{VII})$ 的去除效率只有 $10.3 \%$, 复合的 NZVI/LDHs 材料在相同条件下对 Re(VII)的去除 效率高达 $91.4 \%$.

丙三醇, 又名甘油，是一种外观呈澄明黏稠液态的 有机物. 由于甘油中含有丰富的羟基官能团, 在吸附反 应中可作为与放射性元素铀的反应位点, 因此一些研究 人员试图用甘油对 LDHs 进行改性来提高单体 LDHs 的 吸附性能.例如, Zou 等 ${ }^{[68]}$ 利用水热法合成甘油改性的 Gl-Ca-Al-LDHs 和 Gl-Ni-Al-LDHs, 两种复合材料均用 

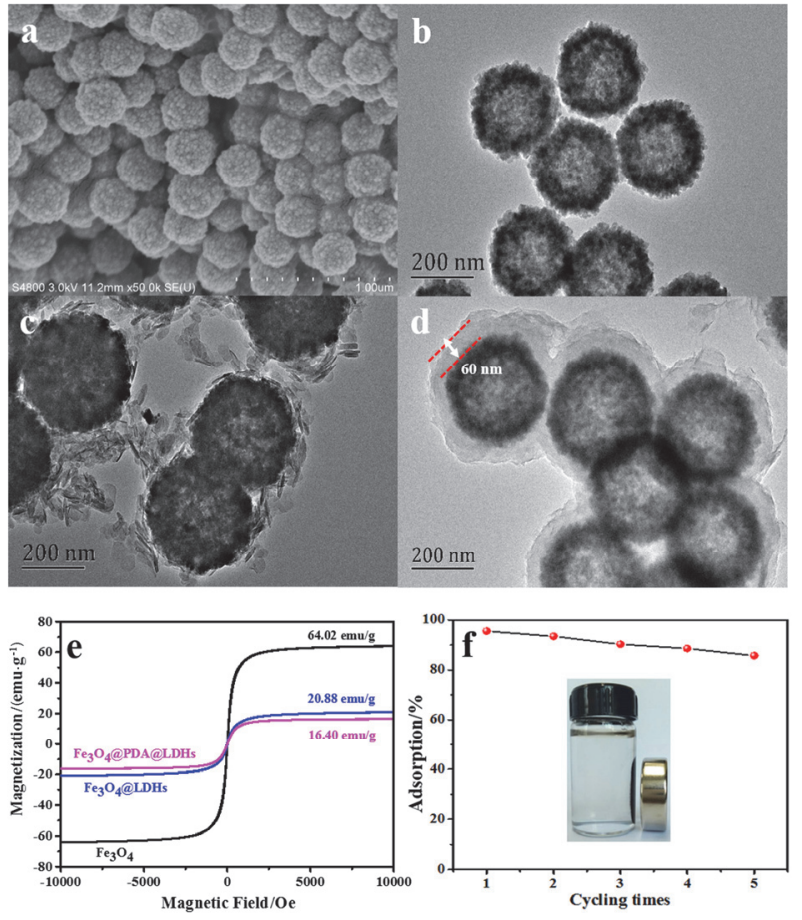

图 $4 \mathrm{Fe}_{3} \mathrm{O}_{4}$ 的 SEM 图 (a) 和 TEM 图 (b); $\mathrm{Fe}_{3} \mathrm{O}_{4} @ \mathrm{LDHs}(\mathrm{c})$ 和 $\mathrm{Fe}_{3} \mathrm{O}_{4} @ \mathrm{PDA} @ \mathrm{LDHs}$ (d) 的 TEM 图 ; $\mathrm{Fe}_{3} \mathrm{O}_{4}, \mathrm{Fe}_{3} \mathrm{O}_{4} @ \mathrm{LDHs}$ 和 $\mathrm{Fe}_{3} \mathrm{O}_{4} @ \mathrm{PDA} @ \mathrm{LDHs}$ 的磁滞回线曲线(e); $\mathrm{Fe}_{3} \mathrm{O}_{4} @ \mathrm{PDA} @ \mathrm{LDHs}$ 对 U(VI) 吸附的循环性(f) ${ }^{[66]}$

Figure 4 SEM (a) and TEM (b) images of $\mathrm{Fe}_{3} \mathrm{O}_{4}$; TEM images of $\mathrm{Fe}_{3} \mathrm{O}_{4} @$ QLDHs (c) and $\mathrm{Fe}_{3} \mathrm{O}_{4} @$ PDA@LDHs (d); VSM profiles (e) of $\mathrm{Fe}_{3} \mathrm{O}_{4}, \mathrm{Fe}_{3} \mathrm{O}_{4} @$ LDHs and $\mathrm{Fe}_{3} \mathrm{O}_{4} @$ PDA@LDHs; Recycle property of $\mathrm{Fe}_{3} \mathrm{O}_{4} @$ PDA@LDHs for U(VI) adsorption from aqueous solutions (f $)^{[66]}$

于水溶液中 $\mathrm{U}(\mathrm{VI})$ 的去除, 并都展现了良好的吸附性能. 在室温条件下, Gl-Ni-Al-LDHs 对 U(VI)的最大吸附量为 $142.3 \mathrm{mg} / \mathrm{g}$, Gl-Ca-Al-LDHs 由于具有更多的官能团以及 大量“ $\mathrm{Ca}-\mathrm{O}$ ”键, 因而对 $\mathrm{U}(\mathrm{VI})$ 具有更好的吸附性能, 最
大吸附量高达 $266.5 \mathrm{mg} / \mathrm{g}$.

综上所述，通过对 LDHs 进行裧烧、插层或者与其 他材料复合，会减少团聚现象，提高材料分散性，使得 改性后的材料具有更大的比表面积和更多的表面官能 团. 因此, LDHs 及其改性材料被越来越多的研究人员 应用于环境中放射性元素铀的去除领域.

\section{4 层状双金属氢氧化物在铀污染控制化学领域 的应用}

为了减少放射性元素铀对生态环境以及人类健康 的危害, 深入了解 LDHs 与 $\mathrm{U}(\mathrm{VI})$ 之间的作用机理是十 分必要的.一般来说, LDHs 和放射性元素之间的作用 机理包括: 静电吸引、离子交换、表面共沉淀以及表面 络合 ${ }^{[69 \sim 73]}$. 在实际自然环境中, 多种去除机理通常共同 发挥作用. 表 1 总结了不同的 LDHs 材料与 U(VI)之间 的相互作用机理. 不同的 LDHs 材料对 U(VI)表现出了 不同的吸附能力, 这与环境条件(如溶液 $\mathrm{pH}$ 、离子强度、 温度、反应时间等)、吸附质性质以及吸附剂的结构性 质相关. 由于相关的报道及系统综述较为详尽, 因此本 文不在赘述. 本综述主要从光谱分析方面对层状双金属 氢氧化物与放射性元素铀之间的作用机理进行分析研 究.

光谱分析技术可以深入探测 $\mathrm{LDHs}$ 与放射性元素铀 之间的微观作用机理. 下面, 本文详细介绍傅里叶变换 红外光谱(FTIR)、X 射线衍射光谱(XRD)、X 射线光电 子能谱(XPS)和扩展 X 射线吸收精细结构谱(EXAFS)在 铀污染去除机理方面的具体应用.

\section{1 傅里叶变换红外光谱(FTIR)}

傅里叶变换红外光谱(Fourier transformed infrared spectroscopy, FTIR)可根据不同物质选择性吸收红外光

表 1 层状双金属氢氧化物及其复合材料对 U(VI)的去除及作用机理

Table 1 The removal of U(VI) by layered double hydroxides and their composites and interaction mechanisms

\begin{tabular}{|c|c|c|c|c|c|c|c|}
\hline 吸附剂 & 污染物 & $m / V(\mathrm{~g} / \mathrm{L})$ & $\mathrm{pH}$ & $T / \mathrm{K}$ & $Q_{\max } /\left(\mathrm{mg} \cdot \mathrm{g}^{-1}\right)$ & 作用机理 & 文献 \\
\hline Ca-Mg-Al-LDH & $\mathrm{U}(\mathrm{VI})$ & 0.1 & 5.0 & 298 & 132.5 & 表面配位、静电作用 & {$[58]$} \\
\hline Ca-Mg-Al-LDO 300 & $\mathrm{U}(\mathrm{VI})$ & 0.1 & 5.0 & 298 & 260.0 & 表面配位、静电作用 & {$[58]$} \\
\hline Ca-Mg-Al-LDO ${ }_{500}$ & $\mathrm{U}(\mathrm{VI})$ & 0.1 & 5.0 & 298 & 486.8 & 表面配位、静电作用 & {$[58]$} \\
\hline LDO & $\mathrm{U}(\mathrm{VI})$ & 0.1 & 5.0 & 298 & 237.6 & 表面配位、沉淀和离子交换 & {$[74]$} \\
\hline $\mathrm{LDO} /$ carbon dots & $\mathrm{U}(\mathrm{VI})$ & 0.1 & 5.0 & 298 & 354.2 & 表面配位、沉淀和离子交换 & {$[74]$} \\
\hline GO@LDH & $\mathrm{U}(\mathrm{VI})$ & 0.22 & 4.5 & 298 & 160.0 & 内层配位和静电作用 & {$[16]$} \\
\hline Ca-Al-LDH & $\mathrm{U}(\mathrm{VI})$ & - & 4.0 & 298 & 54.8 & 离子交换 & {$[52]$} \\
\hline $\mathrm{SiO}_{2} @ \mathrm{LDH}$ & $\mathrm{U}(\mathrm{VI})$ & 0.3 & 5.0 & 298 & 303.1 & 表面配位 & {$[21]$} \\
\hline Ca-Al-LDH-Gl & $\mathrm{U}(\mathrm{VI})$ & 0.1 & 5.0 & 298 & 266.5 & 外层配位和静电作用 & {$[68]$} \\
\hline Ni-Al-LDH-Gl & $\mathrm{U}(\mathrm{VI})$ & 0.1 & 5.0 & 298 & 142.3 & 内层配位和静电作用 & {$[68]$} \\
\hline Ca-Al-LDH@CNTs & $\mathrm{U}(\mathrm{VI})$ & 0.1 & 6.0 & 298 & 382.8 & 表面配位和静电作用 & {$[38]$} \\
\hline $\mathrm{Fe}_{3} \mathrm{O}_{4} @ \mathrm{PDA} @ \mathrm{LDHs}$ & $\mathrm{U}(\mathrm{VI})$ & 0.15 & 5.0 & 298 & 344.0 & 表面配位 & {$[66]$} \\
\hline $\operatorname{CaTiO} x$ & $\mathrm{U}(\mathrm{VI})$ & 0.1 & 5.0 & 293 & 241.7 & 内层配位 & {$[51]$} \\
\hline $\mathrm{CaAlO} x$ & $\mathrm{U}(\mathrm{VI})$ & 0.1 & 5.0 & 293 & 258.3 & 内层配位 & {$[51]$} \\
\hline
\end{tabular}


区的电磁辐射对高分子有机材料进行结构分析 ${ }^{[2,75,76]}$. 傅里叶变换红外光谱可对低浓度样品快速测定, 不会破 坏试样结构, 测定结果较准确. 另外, 红外光谱可在光 密度 $\geqslant 10^{-5} \mu \mathrm{mol}$ 的浓度范围对样品进行测定, 但部分 物质不存在红外分析活性, 因此不能被检测到 ${ }^{[77,78]}$. 如 图 5(a)所示, 对于 Ca-Al-LDH-Gl, 羟基(-OH)的伸缩振 动峰位于约 $3443 \mathrm{~cm}^{-1}$, 而位于 $2515 \mathrm{~cm}^{-1}$ 处的吸收峰则 归属于 $\mathrm{C}-\mathrm{H}$ 键的伸缩振动, 主要是由于甘油分子脱水 造成的. 同时, Ni-Al-LDH-Gl 的 FTIR 光谱中于 2185 $\mathrm{cm}^{-1}$ 处出现一个新的吸收峰 $(\mathrm{C}=\mathrm{N}$ 键 $)$, 产生于水溶液 中的甘油, $\mathrm{CO}_{3}{ }^{2-}$ 和 $\mathrm{NO}_{3}{ }^{-}$的交联作用, 进一步表明甘油 分子被成功嫁接到 LDHs 表面 ${ }^{[68]}$.

\section{$4.2 X$ 射线衍射光谱(XRD)}

$X$ 射线衍射光谱(X-ray diffraction, XRD)是通过对 样品进行 $X$ 射线衍射, 获取被测样品内部分子或原子结 构形态等微观信息的一种方法 ${ }^{[79,80]}$. X 射线衍射主要是 对材料进行定性和定量分析, 定性分析是通过比较被测 样品和标准物相的衍射数据来确定样品中存在的物相 种类, 定量分析是根据衍射强度确定各物相的具体含 量 ${ }^{[81]}$. 如图 5(b)所示, Zou 等 ${ }^{[58]}$ 将制备的 Ca-Mg-Al-LDH 和 Ca-Mg-Al- $\mathrm{LDO}_{x}$ 材料进行了 XRD 分析, 当 $2 \theta=15.2^{\circ}$

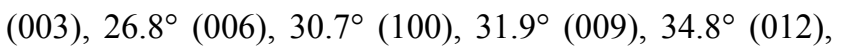
$42.1^{\circ}(015), 45.3^{\circ}(018)$ 和 $52.6^{\circ}$ (1010)均对应为 Ca-MgAl-LDH 的特征峰. 此外, 峰尖锐且对称, 说明 Ca-MgAl-LDH 材料具有很高的结晶度和纯度 ${ }^{[54,82]}$. 另外,
Ca-Mg-Al-LDH 和 Ca-Mg-Al-LDO $x$ 对 U(VI)的吸附动力 学以及吸附机理如图 5(c)所示.

但是，直接使用 XRD 光谱分析方法存在一定局限 性. 由于同族元素具有相似的晶体结构和化学性质，易 在相同位置出现衍射峰，导致无法确定物相. 因此对于 组成元素未知的被测样品需要先进行元素的定性分析.

\section{$4.3 X$ 射线光电子能谱(XPS)}

$\mathrm{X}$ 射线光电子能谱(X-ray photoelectron spectroscopy，XPS)是用 X 射线辐射被测样品，使价电子或内层电 子受激发发生跃迁 ${ }^{[83,84]}$, 以光电子的动能 $\left(E_{\mathrm{b}}=\mathrm{h} v\right.$ 光能 量 $-E_{\mathrm{k}}$ 动能 $-\mathrm{W}$ 功函数)为横坐标, 相对强度(脉冲/ $\mathrm{s}$ )为 纵坐标可做出光电子能谱图, 进而推测样品中所含元素 种类及元素价态等信息 ${ }^{[85,86]}$. XPS 可以用来测量：(1)可 以根据能谱图中特征谱线的位置对元素进行定性分析 (除 H、He 以外). (2)根据能谱图中光电子谱线强度对元 素进行定量分析. (3)测定被测样品表面的化学组成或元 素组成，原子价态和表面能态分布. (4)精确测量内层电 子结合能的化学位移, 提供化学键以及电荷分布等信 息. XPS 的不足之处是只能检测原子浓度在 $0.05 \%$ $1.0 \%$ 之间的物质，而且必须在真空条件下测定，这可能 改变被测样品的某些性质 ${ }^{[87 ~ 89]}$. 如图 6 所示, Yang 等 ${ }^{[66]}$ 发现磁性多巴胺外覆分层水滑石材料 $\left(\mathrm{Fe}_{3} \mathrm{O}_{4}-\mathrm{PDA}-\right.$ LDHs)吸附 U(VI)之后的 C1s、N1s 和 O1s 的 XPS 能谱 都由较低的结合能转移到较高的结合能, 说明含碳、氮、 氧的官能团在整个吸附过程中占主要作用.
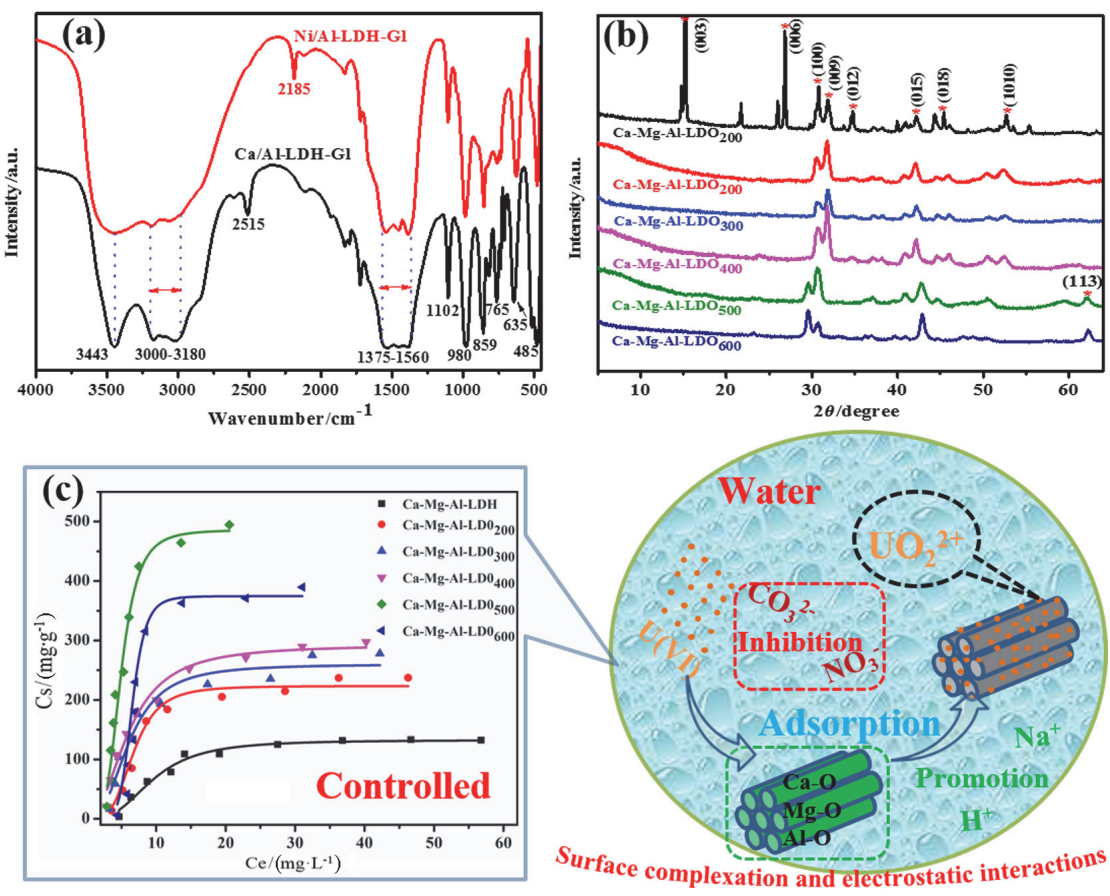

图 5 (a) Ca-Al-LDH-Gl 和 Ni-Al-LDH-Gl 材料的 FTIR 光谱对比图 ${ }^{[68]}$; (b) Ca-Mg-Al-LDH 和 Ca-Mg-Al-LDO $x$ 的 XRD 光谱图 ${ }^{[58]}$; Ca-Mg-Al-LDH 和 Ca-Mg-Al-LDOx 复合材料对 U(VI) 的吸附动力学及相互作用机理 ${ }^{[8]}$

Figure 5 (a) FT-IR spectra of as-prepared Ca-Al-LDH-Gl and Ni-Al-LDH-Gl samples ${ }^{[68]}$; (b) XRD patterns of Ca-Mg-Al-LDH and Ca-Mg-Al-LDOx samples $^{[58]}$; (c) Adsorption kinetics and interaction mechanism of U(VI) on Ca-Mg-Al-LDH and Ca-Mg-Al-LDOx $x^{[58]}$ 

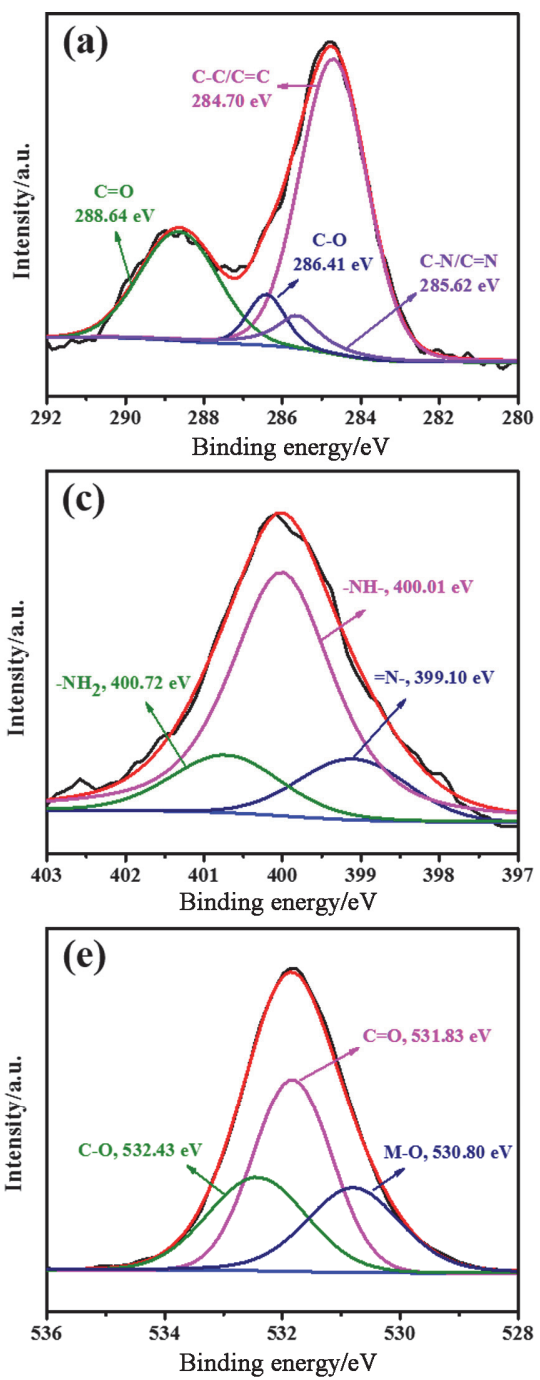
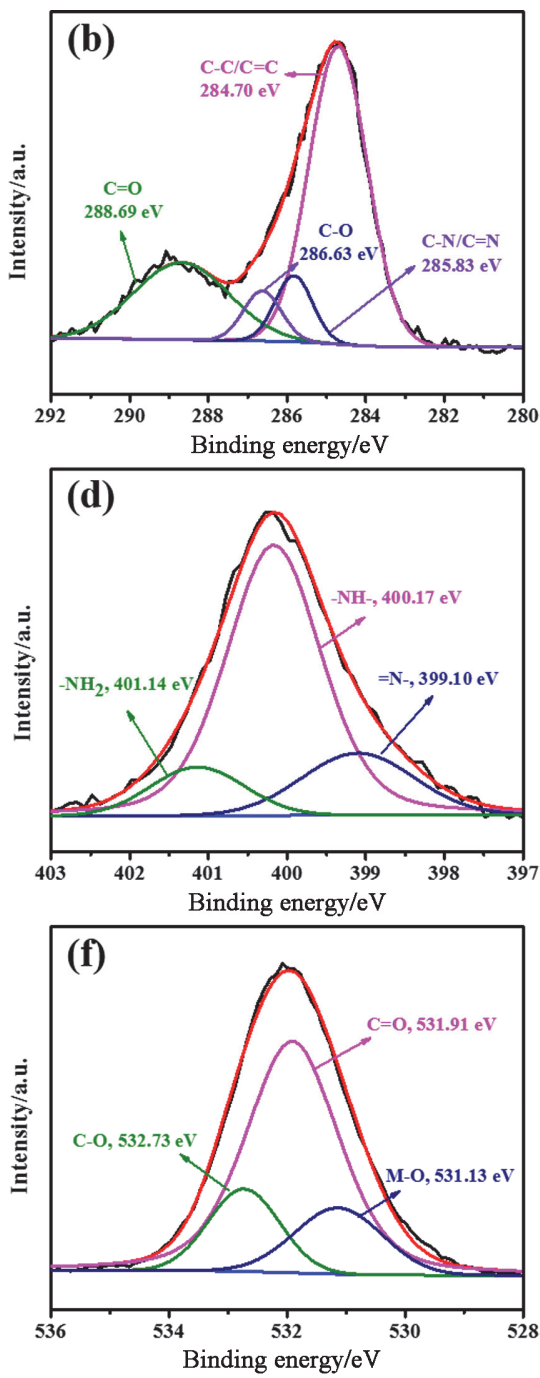

图 $6 \mathrm{Fe}_{3} \mathrm{O}_{4}-\mathrm{PDA}-\mathrm{LDHs}$ 纳米材料的 (a) $\mathrm{C} 1 \mathrm{~s},(\mathrm{c}) \mathrm{N} 1 \mathrm{~s}$ 和(e) $\mathrm{O}$ 1s 光谱以及吸附 $\mathrm{U}(\mathrm{VI})$ 之后的 (b) $\mathrm{C} 1 \mathrm{~s},(\mathrm{~d}) \mathrm{N} 1 \mathrm{~s}$ 和(f) $\mathrm{O}$ 1s 光谱 ${ }^{[66]}$

Figure 6 Deconvolution analysis of (a) C 1s, (c) N 1s, and (e) O 1s XPS spectra of $\mathrm{Fe}_{3} \mathrm{O}_{4}$-PDA-LDHs and (b) C 1s, (d) N 1s, and (f) O 1s XPS spectra of $\mathrm{Fe}_{3} \mathrm{O}_{4}-\mathrm{PDA}-\mathrm{LDHs}+\mathrm{U}(\mathrm{VI})^{[66]}$

\section{4 扩展 X 射线吸收精细结构谱}

扩展 X 射线吸收精细结构谱(extended X-ray absorption fine structure, EXAFS) 是 $\mathrm{X}$ 射线吸收限高能侧 $30 \sim 1000 \mathrm{eV}$ 范围内吸收系数随入射 $\mathrm{X}$ 光子能量增加而 起伏振荡的现象, 近年来它被广泛应用于测定多原子气 体和凝聚态物质吸收原子周围的局域结构, 成为结构分 析的一种新技术 ${ }^{[00]}$. 通过 EXAFS 分析技术可以研究指 定元素原子的近邻结构, 可测配位原子的种类、个数以 及原子间距等 ${ }^{[36,91]}$. EXAFS 方法的四大优势: (1)样品制 备简单, 能同时适用于晶体和非晶体材料的研究. (2)各 个元素互不干扰, 对不同元素的原子, 可由吸收边位置 不同分别进行研究. (3)浓度很低的样品, 百万分之几的

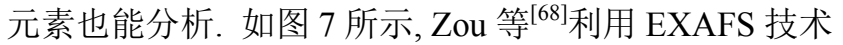
系统地分析了 $\mathrm{pH}=5$ 时 Ca-Al-LDH-Gl 和 Ni-Al-LDH-Gl 吸附 $\mathrm{U}(\mathrm{VI})$ 的 $k^{2}$-加权的 $\chi(k)$ 光谱以及其径向结构函数图. Ni-Al-LDH-Gl-U(VI)轴向上的 2 个氧原子 $\left(\mathrm{O}_{\mathrm{ax}}\right)$ 和赤道面
上的 6 个氧原子 $\left(\mathrm{O}_{\mathrm{eq}}\right)$ 分别位于约 $1.82 \AA$ 和约 $2.41 \AA$ 的 位置, 即 $\mathrm{U}^{-} \mathrm{O}_{\mathrm{ax}}$ (约 $1.82 \AA$ ) 以及 $\mathrm{U}-\mathrm{O}_{\mathrm{eq}}$ (约 $2.41 \AA$ ) 壳层显 示的是 $\mathrm{UO}_{2}{ }^{2+}$ 的距离. 同样地, Ca-Al-LDH-Gl-U(VI)的 EXAFS 图谱表现出相同的趋势, 说明两种吸附剂在吸 附过程中都没有发生元素价态的变化 ${ }^{[92,93]}$. 两种吸附剂 中位于约 $2.6 \AA, 3.0 \AA$ 和 $3.1 \AA$ 位置的特征峰分别对应的 是 U-Al (约 $3.3 \AA$ ), U-Ni (约 $3.6 \AA$ ) 和 U-Ca (约 $3.7 \AA$ ), 说 明 $\mathrm{U}(\mathrm{VI})$ 在两种 $\mathrm{LDHs}$ 材料的氧壳上形成了内层表面配 合物，极大地促进了吸附过程的进行.

以上四种光谱分析手段被广泛用于层状双金属氢 氧化物及其复合材料对放射性元素铀去除机理的研究, 同时使用多种光谱分析方法可以减小分析误差，获得更 准确的实验结果.

\section{5 展望}

层状双金属氢氧化物及其复合材料由于其表面性 

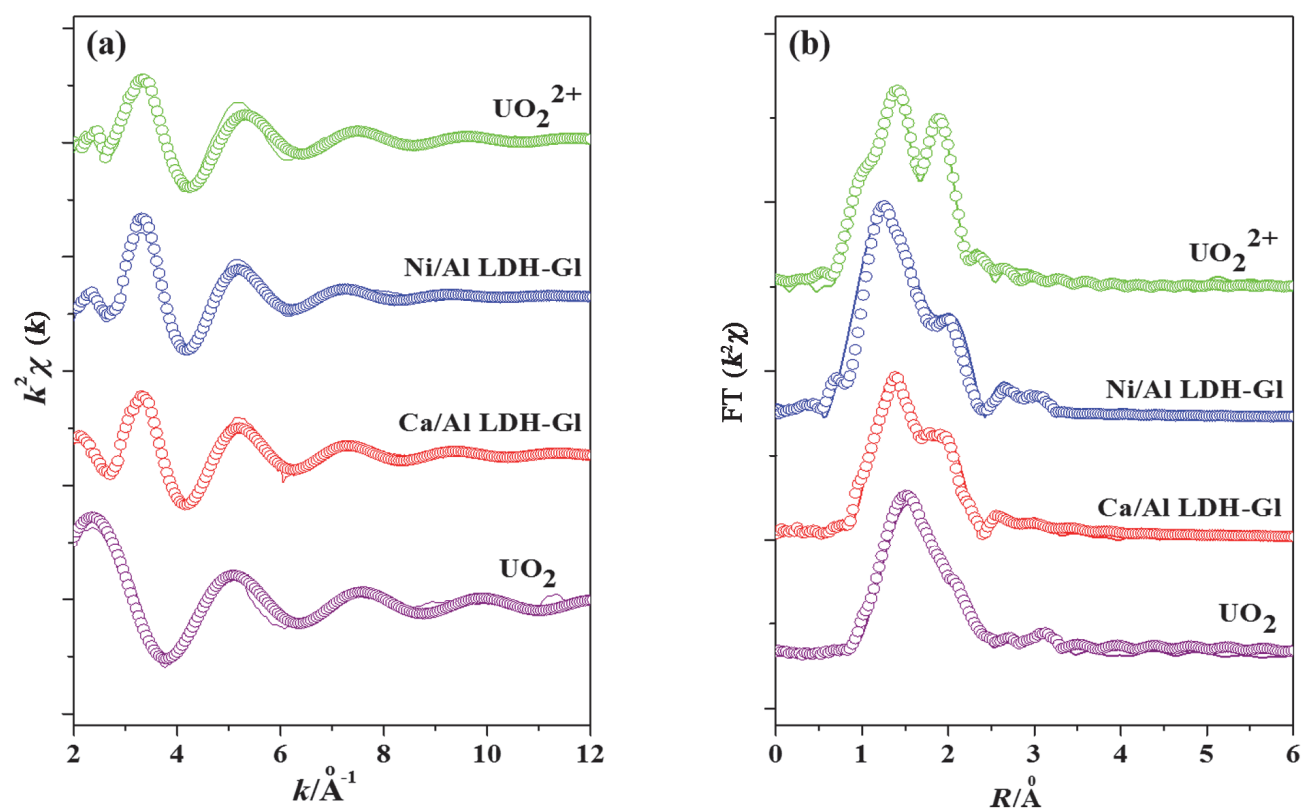

图 7 标准样品和吸附样品的 $k^{2}$-加权的 $\chi(k)$ 光谱(a) 以及其径向结构函数(b $)^{[68]}$

Figure $7 \mathrm{U} \mathrm{L}_{\mathrm{III}}$-edge background subtracted, $k^{2}$-weighted $\chi(k)$ data (a) and corresponding Fourier transformed EXAFS spectra (b) of standards $\left(\mathrm{UO}_{2}{ }^{2+}\right.$ or $\mathrm{UO}_{2}$ ) and adsorption samples ${ }^{[68]}$

表 2 四种光谱分析技术的优缺点分析

Table 2 The advantages and disadvantages of four spectral analysis techniques

\begin{tabular}{|c|c|c|}
\hline 光谱分析方法 & 优点 & 缺点 \\
\hline $\begin{array}{l}\text { 傅里叶变换红外光谱 } \\
\text { (FTIR) }\end{array}$ & $\begin{array}{l}\text { 样品浓度要求低; 测定结果特征性较强, 不破坏样品结构; } \\
\text { 灵敏度高 }\end{array}$ & 部分物质不具有红外分析活性, 无法检测 \\
\hline $\begin{array}{l}X \text { 射线衍射光谱 } \\
\text { (XRD) }\end{array}$ & 可对样品进行定量和定性分析 & $\begin{array}{l}\text { 对于组成元素未知的被测样品必须先进行元素定性分析, 避免 } \\
\text { 同族元素在相同位置出现衍射峰 }\end{array}$ \\
\hline $\begin{array}{l}X \text { 射线光电子能谱 } \\
\text { (XPS) }\end{array}$ & $\begin{array}{l}\text { 测定被测样品表面化学组成, 原子价态和表面能态分布 } \\
\text { (除 } \mathrm{H} 、 \mathrm{He} \text { 以外); 定量分析未知组分组成; 表面灵敏度高 }\end{array}$ & $\begin{array}{l}\text { 样品原子浓度的检测范围必须在 } 0.05 \% \sim 1.0 \% \text { 之间; 测定条件 } \\
\text { 必须为真空, 可能改变样品的某些性质 }\end{array}$ \\
\hline $\begin{array}{l}\text { 扩展 X 射线吸收精细 } \\
\text { 结构谱(EXAFS) }\end{array}$ & $\begin{array}{l}\text { 可测指定元素原子的近邻结构, 配位原子的种类、个数以 } \\
\text { 及原子间距等参量; 样品制备简单, 同时适用晶体与非晶 } \\
\text { 体材料研究; 各个元素检测互不干扰 }\end{array}$ & $\begin{array}{l}\text { 不能区分原子序数几乎没有差异的散射原子; 无法准确区分桥 } \\
\text { 接多核中心处 }>3 \AA \text { 的峰; 不能区分存在于本体溶液或矿物孔 } \\
\text { 中的络合物 }\end{array}$ \\
\hline
\end{tabular}

质和官能团的共同作用对放射性元素铀具有很好的去 除效果，而且来源广泛、制备简单以及易回收利用，因 此近年来被广泛研究与应用. 本文总结了近年来 LDHs 材料在铀污染处理领域的研究进展, 详细介绍了 LDHs 材料的合成和改性方法, 并从光谱分析方面对 LDHs 与 U(VI)的作用机理进行了深入探讨. 尽管 LDHs 材料在 吸附领域反响巨大, 但是也面临着诸多挑战. (1)大规模 化生产 LDHs 材料的技术仍需要进一步探索. (2)需要寻 找更好的改性材料以及更优良的改性方法以提高其对 污染物的选择吸附能力. (3)目前 LDHs 的应用还处于实 验室研究阶段, 距离实际应用仍然有很长一段路. 随着 工业的迅猛发展以及日渐受到重视的环保工作, 越来越 多的科研工作者将投身到环境污染处理领域中来, 因此 多功能的 LDHs 材料在环境污染物处理领域的发展前景 十分广阔.

\section{作者简介}

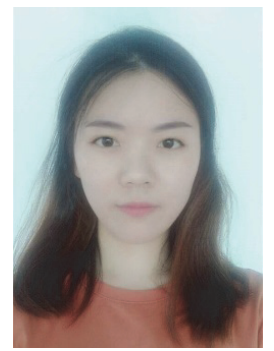

王宁, 女, 汉族, 硕士, 南华大学与华北电力大学联合培 养硕士研究生. 主要从事纳米复合材料吸附环境污染物的研 究. 


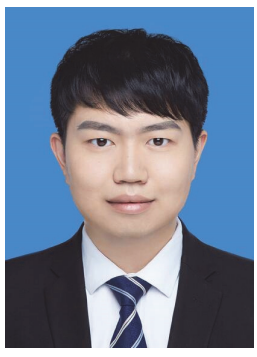

庞宏伟, 男, 汉族, 硕士, 华北电力大学硕士研究生. 主 要从事纳米复合材料吸附铀的研究.

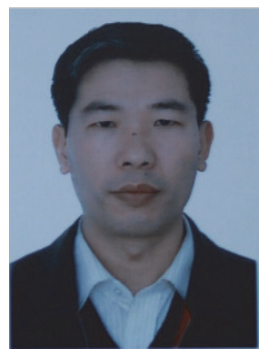

王宏青, 男, 教授, 南华大学化学化工学院院长, 硕士研 究生导师, 澳大利亚 Monash University 的访问学者, 中国核学 会铜系化学会理事, 中国核学会(化学会)核化学与放射化学分 会环境放射化学专业委员会委员, 湖南省化学化工学会副理 事长, 湖南省盐卤化工联盟副理事长, 国际著名刊物 J. Hazard. Mater、Talanta、Synlett、Bioorg. Med.Chem. Lett、J. Mol. Struct. Spectrochim. Acta. A 等审稿专家. 主要从事功能分子 的设计、合成和性质以及分子识别分离研究. 主持国家自然基 金、湖南省省市联合基金、湖南自然科学基金、湖南省教育 厅重点与青年项目等项目 6 项; 获衡阳市科技进步二等和三 等奖各 1 项. 在国内外学术期刊等发表学术论文 70 余篇, 其 中被 SCI 收录 40 余篇, EI 收录 3 篇, 获得发明专利 3 件, 出版 专著一部, 编写出版教材一部, 指导研究生获得湖南省优秀 硕士论文 1 人.

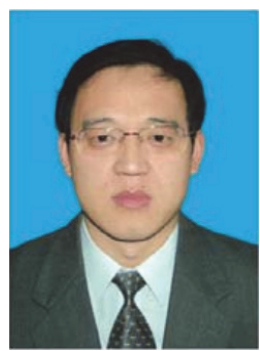

王祥科, 男, 教授, 博士生导师, 2003 年入选中科院百人 计划引进海外杰出人才, 2012 年获国家杰出青年科学基金, 2015 年被评为教育部长江学者特聘教授, 2016 年入选科技部 中青年科技创新领军人才和 2016 年北京市百名科技领军人才 称号, 2017 年第三批国家“万人计划”领军人才称号等. 2014 年调入华北电力大学, 现在为环境科学与工程学院院长, 主 要从事三废治理、纳米材料在废水处理、等离子体技术应用、 环境污染检测和治理中的应用等方面的研究工作. 主持与参 加了中科院百人计划项目、国家自然科学基金杰出青年科学 基金、重点基金、国家 973 项目等多项研究发表论文 330 余 篇, 有 80 多篇论文被评为高引用论文(highly cited paper)和热 点论文(hot paper), $\mathrm{H}$ 影响因子为 93 , 被他人正面引用和评价
24000 多次；2014～2018 年连续五年被汤森路透/科点唯安评 选为高被引科学家, 同时入选工程技术、环境科学与生态学 两个领域; 受邀请担任多个国内外学术期刊, 如 J. Hazard. Mater、Sci. China Chem、 J. Mol. Liquid、Radiochim. Acta、《中 国科学》化学期刊编委和多个学术委员会委员.

\section{References}

[1] Yin, L.; Song, S.; Wang, X. X.; Niu, F. L.; Ma, R.; Yu, S. J.; Wen, T.; Chen, Y. T.; Hayat, T.; Alsaedi, A.; Wang, X. K. Environ. Pollut. 2018, 238, 725 .

[2] Pang, H. W.; Wang, X. X.; Yao, W.; Yu, S. J.; Wang, X. K. Sci. Sinica Chim. 2018, 48, 58. (庞宏伟, 王祥学, 姚文, 于淑君, 王祥科, 中国科学: 化学, 2018, 48, 58.)

[3] Liang, Y.; Gu, P.; Yao, W.; Yu, S.; Wang, J.; Wang, X. Prog. Chem. 2017, 29, 1062. (梁宇, 顾鹏程, 姚文, 于淑君, 王建, 王祥科, 化 学进展, 2017, 29, 1062.)

[4] Sun, Y.; Wu, Z. Y.; Wang, X.; Ding, C.; Cheng, W.; Yu, S. H.; Wang, X. Environ. Sci. Technol. 2016, 50, 4459.

[5] Chen, H. J.; Huang, S. Y.; Zhang, Z. B.; Liu, Y. H.; Wang, X. K. Acta Chim. Sinica 2017, 75, 560. (陈海军, 黄舒怡, 张志宾, 刘云海, 王祥科, 化学学报, 2017, 75, 560.)

[6] Yang, D. X.; Wang, X. X.; Song, G.; Zhao, G. X.; Chen, Z.; Yu, S. J.; Gu, P. C.; Wang, H. Q.; Wang, X. K. Sci. Bull. 2017, 62, 1609.

[7] Sun, Y.; Ding, C.; Cheng, W.; Wang, X. J. Hazard. Mater. 2014, 280, 399.

[8] Ding, C.; Cheng, W.; Sun, Y.; Wang, X. Geochim. Cosmochim. Acta $\mathbf{2 0 1 5}, 165,86$.

[9] Yang, S. Y.; Wang, X. X.; Chen, Z. S.; Li, Q.; Wei, B. B.; Wang, X. K. Prog. Chem. 2018, 30, 225. (杨姗也, 王祥学, 陈中山, 李倩, 韦坢特, 王祥科, 化学进展, 2018, 30, 225.)

[10] Zhang, T.; Gregory, K.; Hammack, R. W.; Vidic, R. D. Environ. Sci. Technol. 2014, 48, 4596.

[11] Chen, H.; Wang, X.; Li, J.; Wang, X. J. Mater. Chem. A 2015, 3, 6073.

[12] Nigiz, F. U.; Ozkoc, G.; Hilmioglu, N. D. Mater. Design 2015, 88, 942 .

[13] Zhang, S.; Li, J.; Wang, X.; Huang, Y.; Zeng, M.; Xu, J. ACS Appl. Mater. Inter. 2014, 6, 22116.

[14] Montaña, M.; Camacho, A.; Serrano, I.; Devesa, R.; Matia, L.; Vallés, I. J. Environ. Radioactiv. 2013, 125, 86.

[15] Ma, L.; Wang, Q.; Islam, S. M.; Liu, Y.; Ma, S.; Kanatzidis, M. G. J. Am. Chem. Soc. 2016, 138, 2858.

[16] Yu, S.; Wang, J.; Song, S.; Sun, K.; Li, J.; Wang, X.; Chen, Z.; Wang, X. Sci. China Chem. 2017, 60, 415.

[17] Yin, L.; Wang, P.; Wen, T.; Yu, S.; Wang, X.; Hayat, T.; Alsaedi, A.; Wang, X. Environ. Pollut. 2017, 226, 125

[18] Du, Y.; Wang, J.; Zou, Y.; Yao, W.; Hou, J.; Xia, L.; Peng, A.; Alsaedi, A.; Hayat, T.; Wang, X. Sci. Bull. 2017, 62, 913.

[19] Yao, Y.; He, B.; Xu, F.; Chen, X. Chem. Eng. J. 2011, 170, 82.

[20] Hu, W.; Lu, S.; Song, W.; Chen, T.; Hayat, T.; Alsaedie, A.; Chen, C.; Liu, H. Appl. Clay Sci. 2018, 157, 121.

[21] Yang, D.; Song, S.; Zou, Y.; Wang, X.; Yu, S.; Wen, T.; Wang, H.; Hayat, T.; Alsaedi, A.; Wang, X. Chem. Eng. J. 2017, 323, 143.

[22] Wang, X.; Yu, S.; Wu, Y.; Pang, H.; Yu, S.; Chen, Z.; Hou, J.; Alsaedi, A.; Hayat, T.; Wang, S. Chem. Eng. J. 2018, 342, 321.

[23] Wu, X. L.; Wang, L.; Chen, C. L.; Xu, A.-W.; Wang, X. K. J. Mater Chem. 2011, 21, 17353 .

[24] Sun, Z.; Gu, L.; Zheng, J.; Zhang, J.; Wang, L.; Xu, F.; Lin, C. A. Mater. Lett. 2016, 172, 105.

[25] Song, F.; Hu, X. J. Am. Chem. Soc. 2014, 136, 16481

[26] Li, Y.; Shi, L. W.; Liu, Z. S.; Yang, G. Q. Acta Chim. Sinica 2012, 70, 683. (李燕，施利文，刘志山，杨国庆，化学学报，2012，70, 683.)

[27] Zhou, J. Z.; Wu, Y. Y.; Liu, C.; Orpe, A.; Liu, Q.; Xu, Z. P.; Qian, G. R.; Qiao, S. Z. Environ. Sci. Technol. 2010, 44, 8884.

[28] Zhang, M.; Yao, Q.; Lu, C.; Li, Z.; Wang, W. ACS Appl. Mater. Inter. 2014, 6, 20225.

[29] Tan, X.; Fang, M.; Ren, X.; Mei, H.; Shao, D.; Wang, X. Environ. Sci. Technol. 2014, 48, 13138.

[30] Huang, P. P.; Cao, C. Y.; Wei, F.; Sun, Y. B.; Song, W. G. RSC $A d v$. 2015, 5, 10412 .

[31] Shao, M.; Ning, F.; Zhao, J.; Wei, M.; Evans, D.; Duan, X. J. Am. Chem. Soc. 2012, 134, 1071. 
[32] Zhou, L.; Shao, M.; Wei, M.; Duan, X. J. Energy Chem. 2017, 26, 194.

[33] Peng, F.; Luo, T.; Yuan, Y. New J. Chem. 2014, 38, 4427.

[34] Li, J.; Fan, Q.; Wu, Y.; Wang, X.; Chen, C.; Tang, Z.; Wang, X. J. Mater. Chem. A 2016, 4, 1737.

[35] Wang, X.; Pang, H.; Wu, Y.; Yu, S.; Song, G.; Ma, X.; Xu, P. Sci. Sinica Chim. 2018. (王祥学, 庞宏伟, 吴忆涵, 于淑君, 宋刚, 马 宵颖, 许佩瑶, 中国科学: 化学, 2018.)

[36] Gu, P. C.; Zhang, S.; Li, X.; Wang, X. X.; Wen, T.; Jehan, R.; Alsaedi, A.; Hayat, T.; Wang, X. K. Environ. Pollut. 2018, 240, 493.

[37] Koilraj, P.; Kamura, Y.; Sasaki, K. J. Mater. Chem. A 2018, 6, 10008 .

[38] Chen, H.; Che, Z.; Zhao, G.; Zhang, Z.; Xu, C.; Liu, Y.; Chen, J.; Zhuang, L.; Haya, T.; Wang, X. J. Hazard. Mater. 2018, 347, 67.

[39] Morel-Desrosiers, N.; Pisson, J.; Israëli, Y.; Taviot-Guého, C.; Besse, J. P.; Morel, J. P. J. Mater. Chem. 2003, 13, 2582.

[40] Li, J.; Fan, Q.; Wu, Y.; Wang, X.; Chen, C.; Tang, Z.; Wang, X. J. Mater. Chem. A 2016, 4, 1737.

[41] Wang, J.; Li, Y.; Chen, W.; Peng, J.; Hu, J.; Chen, Z.; Wen, T.; Lu, S.; Chen, Y.; Hayat, T.; Ahmad, B.; Wang, X. Chem. Eng. J. 2016, $309,445$.

[42] Wang, W.; Zhou, J.; Achari, G.; Yu, J.; Cai, W. Colloid. Surfaces A 2014, 457, 33.

[43] Jaiswal, A.; Mani, R.; Banerjee, S.; Gautam, R.; Chattopadhyaya, M. J. Mol. Liq. 2015, 202, 52.

[44] Wu, X.; Du, Y.; An, X.; Xie, X. Catal. Commun. 2014, 50, 44

[45] Yu, H.; Xu, B.; Bian, L.; Gao, H. J. Syn. Cryst. 2010, 39, 1292. (于 洪波, 徐冰, 边令喜, 高宏, 人工晶体学报, 2010, 39, 1292.)

[46] Yu, S.; Wang, X.; Pang, H.; Zhang, R.; Song, W.; Fu, D.; Hayat, T.; Wang, X. Chem. Eng. J. 2017, 333, 343.

[47] Yu, S.; Liu, Y.; Ai, Y.; Wang, X.; Zhang, R.; Chen, Z.; Chen, Z.; Zhao, G.; Wang, X. Environ. Pollut. 2018, 242, 1.

[48] Siebecker, M.; Li, W.; Syed, K.; Sparks, D. Nat. Commun. 2014, 5, 5003.

[49] Li, W.; Livi, K.; Xu, W.; Siebecker, M.; Wang, Y.; Phillips, B.; Sparks, D. Environ. Sci. Technol. 2012, 46, 11670.

[50] Yao, W.; Yu, S.; Wang, J.; Zou, Y.; Lu, S.; Ai, Y.; Alharbi, N. S.; Alsaedi, A.; Hayat, T.; Wang, X. Chem. Eng. J. 2017, 307, 476.

[51] Hu, Y.; Wang, X.; Zou, Y.; Wen, T.; Wang, X.; Alsaedi, A.; Hayat, T.; Wang, X. Chem. Eng. J. 2017, 316, 419.

[52] Li, Y.; Wang, J.; Li, Z.; Liu, Q.; Liu, J.; Liu, L.; Zhang, X.; Yu, J. Chem. Eng. J. 2013, 218, 295.

[53] Asiabi, H.; Yamini, Y.; Shamsayei, M. J. Hazad. Mater. 2017, 339, 239.

[54] Wu, X.; Tan, X.; Yang, S.; Wen, T.; Guo, H.; Wang, X.; Xu, A. Water Res. 2013, 47, 4159.

[55] Wang, J.; Wang, X.; Tan, L.; Chen, Y.; Hayat, T.; Hu, J.; Alsaedi, A.; Ahmad, B.; Guo, W.; Wang, X. Chem. Eng. J. 2016, 297, 106.

[56] Zou, Y.; Wang, X.; Ai, Y.; Liu, Y.; Li, J.; Ji, Y.; Wang, X. Environ. Sci. Technol. 2016, 50, 3658 .

[57] Wang, R. X.; Wen, T.; Wu, X. L.; Xu, A. W. RSC $A d v$. 2014, 4, 21802.

[58] Zou, Y.; Wang, X.; Wu, F.; Yu, S.; Hu, Y.; Song, W.; Liu, Y.; Wang, H.; Hayat, T.; Wang, X. ACS Sustain. Chem. Eng. 2016, 5, 1173.

[59] Wei, M.; Shi, Z.; Evans, D. G.; Duan, X. J. Mater. Chem. 2006, 16, 2102.

[60] Yu, S.; Wang, X.; Chen, Z.; Wang, J.; Wang, S.; Hayat, T.; Wang, X. J. Hazard. Mater. 2017, 321, 111.

[61] Wen, T.; Wu, X.; Tan, X.; Wang, X.; Xu, A. ACS Appl. Mater. Inter. 2013, 5, 3304 .

[62] Qiu, H.; Cui, B.; Li, G.; Yang, J.; Peng, H.; Wang, Y.; Li, N.; Gao, R.; Chang, Z.; Wang, Y. J. Phys. Chem. C 2014, 118, 14929.

[63] Cerff, M.; Morweiser, M.; Dillschneider, R.; Michel, A.; Menzel, K.; Posten, C. Bioresource Technol. 2012, 118, 289.

[64] Yang, X. Y.; Gong, Z. Q.; Zheng, Y. J.; Liu, F. L. J. Functional Mater. 2005, 36, 667. (杨喜云, 龚竹青, 郑雅杰, 刘丰良, 功能材料, $\mathbf{2 0 0 5}, 36,667$.

[65] Luo, J.; Zhang, G.; Xie, N.; Wang, T.; Gu, Y.; Gong, S.; Wang, C. A. IEEE Photonic. Tech. L. 2015, 27, 998.

[66] Yang, D.; Wang, X.; Wang, N.; Zhao, G.; Song, G.; Chen, D.; Liang, Y.; Wen, T.; Wang, H.; Hayat, T. J. Clean. Prod. 2018, 172, 2033

[67] Sheng, G.; Tang, Y.; Linghu, W.; Wang, L.; Li, J.; Li, H.; Wang, X.; Huang, Y. Appl. Catal. B-Environ. 2016, 192, 268.

[68] Zou, Y.; Liu, Y.; Wang, X.; Sheng, G.; Wang, S.; Ai, Y.; Ji, Y.; Liu, Y.; Hayat, T.; Wang, X. ACS Sustain. Chem. Eng. 2017, 5, 3583.

[69] Yu, S.; Wang, X.; Tan, X.; Wang, X. Inorg. Chem. Front. 2015, 2 , 593.

[70] Sun, Y.; Yang, S.; Chen, Y.; Ding, C.; Cheng, W.; Wang, X. Environ. Sci. Technol. 2015, 49, 4255.

[71] Wang, X.; Fan, Q.; Yu, S.; Chen, Z.; Ai, Y.; Sun, Y.; Hobiny, A.; Alsaedi, A.; Wang, X. Chem. Eng. J. 2016, 287, 448.

[72] Gu, P. C.; Song, S.; Zhang, S.; Wei, B. B.; Wen, T.; Wang, X. K. Acta Chim. Sinica 2018, 76, 701. (顾鹏程, 宋爽, 张塞, 韦牲牲, 文 涛, 王祥科, 化学学报, 2018, 76, 701.)

[73] Zhang, C. L.; Li, X.; Chen, Z. S.; Wen, T.; Huang, S. Y.; Hayat, T.; Alsaedi, A.; Wang, X. K. Sci. China Chem. 2018, 61, 281.

[74] Yao, W.; Wang, X. X.; Liang, Y,; Yu, S. J.; Gu, P. C.; Sun, Y. B.; Xu, C.; Chen, J.; Hayat, T.; Alsaedi, A.; Wang, X. K. Chem. Eng. J. 2018, 332, 775 .

[75] Tan, X.; Ren, X.; Chen, C.; Wang, X. Trends. Anal. Chem. 2014, 61, 107.

[76] Yao, W.; Wu, Y.; Pang, H.; Wang, X.; Yu, S.; Wang, X. Sci. China Chem. 2018, 61, 812 .

[77] Müller, K.; Foerstendorf, H.; Brendler, V.; Rossberg, A.; Stolze, K.; Gröschel, A. Chem. Geol. 2013, 357, 75.

[78] Jin, J.; Sun, K.; Liu, W.; Li, S. W.; Peng, X. Q.; Yang, Y.; Han, L. F.; Du, Z. W.; Wang, X. K. Environ. Pollut. 2018, 236, 745.

[79] Sheng, G. D.; Huang, C. C.; Chen, G. H.; Sheng, J.; Ren, X. M.; Hu, B. W.; Ma, J. Y.; Wang, X. K.; Huang, Y. Y.; Alsaedi, A.; Hayat, T. Environ. Pollut. 2018, 233, 125.

[80] Chen, H. J.; Chen, Z.; Zhao, G. X.; Zhang, Z. B.; Xu, C.; Liu, Y. H.; Chen, J.; Zhuang, L.; Hayat, T.; Wang, X. K. J. Hazard. Mater. 2018 , 347,66 .

[81] Yu, S.; Yin, L.; Pang, H.; Wu, Y.; Wang, X.; Zhang, P.; Hu, B.; Chen, Z.; Wang, X. Chem. Eng. J. 2018, 352, 360.

[82] Zhao, J.; Chen, J.; Xu, S.; Shao, M.; Zhang, Q.; Wei, F.; Ma, J.; Wei, M.; Evans, D.; Duan, X. Adv. Funct. Mater. 2014, 24, 2938.

[83] Sheng, G.; Alsaedi, A.; Shammakh, W.; Monaquel, S.; Sheng, J.; Wang, X.; Li, H.; Huang, Y. Carbon 2016, 99, 123.

[84] Zhao, G. X.; Huang, X. B.; Tang, Z. W.; Huang, Q. F.; Niu, F. L.; Wang, X. K. Poly. Chem. 2018, 9, 3562.

[85] Shao, D.; Li, J.; Tan, X.; Yang, Z.; Okuno, K.; Oya, Y. J. Nucl. Mater. 2015, 457, 118.

[86] Liang, Y.; Gu, P. C.; Yao, W.; Yu, S. J.; Wang, J.; Wang, X. K. Prog. Chem. 2017, 29, 1062. (梁宇, 顾鹏程, 姚文, 于淑君, 王建, 王祥 科, 化学进展, 2017, 29, 1062.)

[87] Xu, H.; Li, G.; Li, J.; Chen, C.; Ren, X. J. Mol. Liq. 2016, 213, 58.

[88] Li, J.; Wang, X. X.; Zhao, G. X.; Chen, C. L.; Chai, Z. F.; Alsaedi, A.; Hayat, T.; Wang, X. K. Chem. Soc. Rev. 2018, 47, 2322.

[89] Li, X.; Liu, Y.; Zhang, C. L.; Wen, T.; Zhuang, L.; Wang, X. X.; Song, G.; Chen, D. Y.; Ai, Y. J.; Hayat, T.; Wang, X. K. Chem. Eng. J. 2018, 336, 241 .

[90] Song, W. C.; Wang, X. X.; Chen, Z. S.; Sheng, G. D.; Hayat, T.; Wang, X. K.; Sun, Y. B. Environ. Pollut. 2018, 237, 228.

[91] Yu, S. J.; Wang, X. X.; Yang, S. T.; Sheng, G. D.; Alsaedi, A.; Hayat, T.; Wang, X. K. Sci. China Chem. 2017, 60, 170.

[92] Sheng, G.; Yang, P.; Tang, Y.; Hu, Q.; Li, H.; Ren, X.; Hu, B.; Wang, X.; Huang, Y. Appl. Catal. B-Environ. 2016, 193, 189.

[93] Wu, Y. H.; Pang, H. W.; Yao, W.; Wang, X. X.; Yu, S. J.; Yu, Z. M.; Wang, X. K. Sci. Bull. 2018, 63, 831.

(Cheng, F.) 